\title{
Plasma prolactin concentrations following epileptic and pseudoseizures
}

\author{
WCJ COLLINS, ${ }^{*}$ O LANIGAN, N CALLAGHAN \\ From the Department of Neurology, Regional Hospital, Cork; and Endocrine Laboratory, ${ }^{*}$ Mater \\ Misericordiae Hospital, Dublin, Ireland
}

SUMMARY Serial plasma prolactin levels were measured following eighteen generalised seizures, ten partial seizures and eight pseudoseizures. Prolactin levels were elevated following generalised seizures, but were normal following the other seizure types. Plasma prolactin levels may, therefore, be helpful in differentiating between generalised and pseudoseizures. The optimal time for estimating the prolactin level was 15-20 minutes following the seizure.

It can be difficult clinically to differentiate an epileptic seizure from a pseudoseizure. In the differentiation, the history and an eye witness account are obviously of prime importance. Aids in the diagnosis include routine and sleep deprivation electroencephalography (EEG), ambulatory EEG, telemetry, CT brain scanning and, sometimes, ambulatory cardiac monitoring. However, these are both time consuming and expensive. A simple biochemical marker of a recent seizure would, therefore, be helpful.

Plasma levels of a variety of hormones have been shown to be elevated following seizures induced by electroconvulsive therapy (ECT) in psychiatric patients. $^{1}$ Two studies ${ }^{23}$ have demonstrated that plasma prolactin levels are elevated following spontaneous generalised (tonic-clonic) seizures. Plasma prolactin may, therefore, be a useful biochemical marker of a recent seizure. The aim of this study was to establish the time course of the changes in plasma prolactin following seizures, and to correlate changes in levels with seizure type.

\section{Patients and methods}

Plasma prolactin was estimated following thirty-six seizures in thirty-five patients. All patients were in-patients at the time of the study. They were divided into three groups:-

(a) Generalised seizures. There were eighteen patients [ten male, eight female: mean age

Address for reprint requests: Dr N Callaghan, Dept. of Neurology, Cork Regional Hospital, Cork, Ireland.

Received 27 August 1982 and in revised form 17 November 1982 Accepted 2 December 1982
$30 \cdot 5$ years (range $7-64$ years)]. All of these patients had had grand mal (tonic-clonic) seizures.

(b) Partial seizures. There were ten patients [five male, five female: mean age $40 \cdot 1$ years (range 12-64 years)]. Nine of these patients had had partial seizures with elementary symptomatology, and one had had partial complex seizures.

(c) Pseudoseizures. There were eight patients [seven male, one female: mean age 26.4 years (range $12-38$ years)]. Six patients had, initially, been misdiagnosed as epileptics. Two patients did have co-existent generalised epilepsy, and one of these was studied twice, after a generalised and a pseudoseizure. $\mathrm{He}$ is, therefore, included in Groups (a) and (c).

This allocation of patients was based on, at least, two of the following: clinical assessment, routine EEG monitoring, telementry, psychiatric assessment.

Twenty-nine of the patients were taking anticonvulsant medication at the time of the study. The anticonvulsant drugs used, and the number of patients in each group taking each anticonvulsant drug are illustrated in table 1 . The numbers on

Table 1 Seizure type related to anticonvulsant medication

\begin{tabular}{llll}
\hline Drug & Generalised & Partial & Pseudoseizures \\
\hline Phenytoin & 6 & 7 & 5 \\
Carbamazepine & 7 & 2 & 1 \\
Phenobarbitone & 2 & 4 & 2 \\
Primidone & 2 & - & 1 \\
Sodium valproate & 3 & 1 & 1 \\
Methoin & - & - & 1 \\
\hline
\end{tabular}


Table 2 Seizure-types related to monotherapy and polypharmacy

\begin{tabular}{llll}
\hline & Monotherapy & Polypharmacy & No treatment \\
\hline Generalised & 8 & 5 & 5 \\
Partial & 6 & 4 & $\frac{1}{2}$ \\
Pseudoseizures & 4 & 2 & \\
\hline
\end{tabular}

monotherapy and on polypharmacy are illustrated in table 2.

Plasma prolactin was estimated at $5,15,30,60$, 90,120 , and 180 minutes following seizures. Venous samples were drawn via an intravenous cannula with a heparin lock (except at $5 \mathrm{~min}$ ). Studies were performed at various times throughout the 24 hours. Prolactin was measured using a radioimmunoassay technique, as previously described. ${ }^{4}$ Sample $(50 \mu l)$ was incubated with antibody (VLS No 3 at final dilution $1: 300,000)$ for three days at $4^{\circ} \mathrm{C}$ followed by a further three days incubation with tracer (VLS No 5 , lactoperoxidase iodination with sequential purification on Sephadex G-50 and G-100). Separation was by conventional second antibody. The doseresponse curve was set up using MRC standard 71/ 222. Normal plasma prolactin levels for this laboratory are less than $300 \mathrm{mU} / 1$. Moderately elevated levels (for example 400-450mU/l) may sometimes be caused by physiological factors such as the stress induced by venipuncture. We feel that this is unlikely in our cases as most samples were taken via an intravenous cannula.

\section{Results}

The data consisted of prolactin measurements carried out at the time listed above. The data was converted into three statistics:

(a) AUC Area under the prolactin-time curve calculated using the trapezoidal rule.

(b) Cmax Maximum prolactin level.

(c) Tmax Time of the maximum prolactin level. For each patient an AUC, Cmax and Tmax has been calculated. Each of these statistics was then subjected to a non-parametric analysis of variance, using the Krushal-Wallis technique. If this analysis indicated differences between the three groups of patients, Dunn's multiple comparison technique was applied. Table 3 shows that the tests for AUC and

Table 3 Statistical analysis of results

\begin{tabular}{lcll}
\hline & $A U C(m U / l)$ & $C_{\max }(m U / l)$ & $T_{\text {max }}(\min )$ \\
\hline Generalised & 59698 & 670 & 15 \\
Partial & 28513 & 220 & 15 \\
Pseudoseizures & 25988 & 200 & 22.5 \\
$\chi^{2}$ (Ref 2) & 24.234 & 26.300 & 2.061 \\
$p$ & $<0.001$ & $<0.001$ & $>0.3$ \\
\hline
\end{tabular}

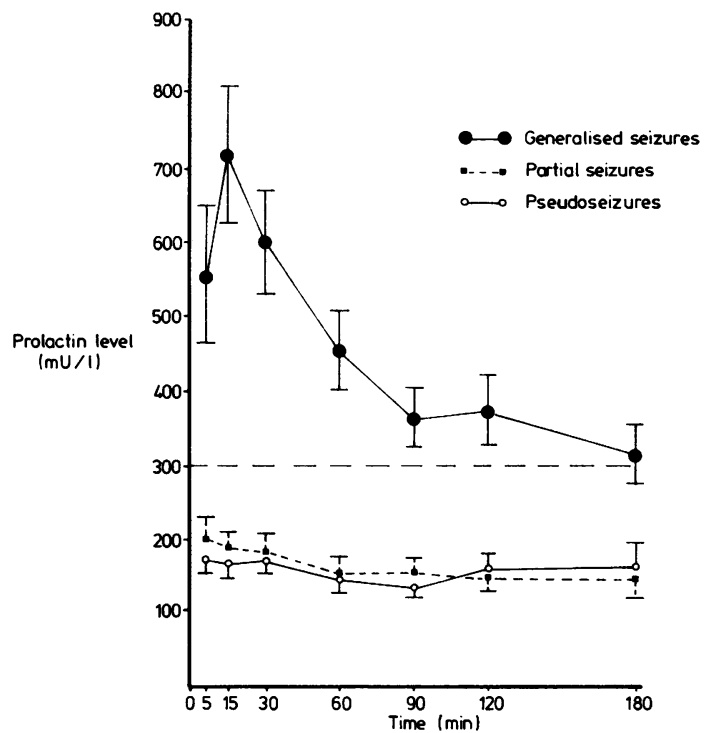

Fig 1. Mean plasma prolactin levels, $\pm S E M$, following generalised, partial and pseudo-seizures.

Cmax were significant, suggesting that there were differences between the groups. The Dunn tests show that the generalised group was different from both the partial seizures and pseudoseizures groups with respect to AUC-prolactin ("available" prolac-

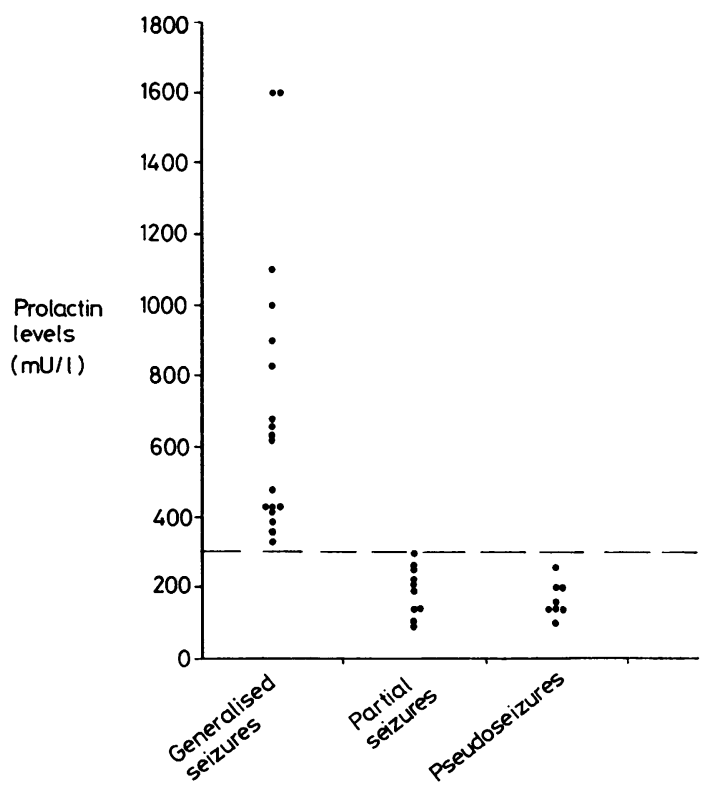

Fig 2 Plasma prolactin level in each patient at $15 \mathrm{~min}$. following the seizure. 
tin) and Cmax, reflecting the fact that the prolactin levels ran generally higher in the generalised than in the other groups. There were no differences for the Tmax, that is although the peaks were different for different groups of patients, they occurred at the same time in all groups, around fifteen - twenty minutes after a seizure.

The arithmetic mean of the prolactin levels at each time of estimation was also calculated for the three groups. These are illustrated in fig 1 . The plasma prolactin levels for each patient, in all three groups, at $15 \mathrm{~min}$ (Tmax) are illustrated in fig 2 . It can be seen that the plasma prolactin level was elevated in all patients in the generalised seizure group and was normal in all of the patients in the other two groups. There was no overlap between the generalised seizure group and either the pseudoseizures or partial seizures group.

We do not have a baseline, that is a preseizure prolactin level for these patients, and it is conceivable that patients with generalised seizures have higher plasma prolactin levels. Certainly, there is a suggestion from our results that three hours after a generalised seizure prolactin levels are still higher than in the other groups.

\section{Discussion}

The results of this study confirm the findings of others ${ }^{23}$ that plasma prolactin is elevated following generalised seizures. The timing of the peak level, around 15-20 minutes after a seizure in this study, is also in agreement with the previous two studies. However, the magnitude of the peak level, usually two - three times above the rormal range in this study, is less than that found in the previous studies. We are, as yet, unable to explain this discrepancy, although, the magnitude and the timing of the peak level in this study are similar to those found in a study of the changes in plasma prolactin following ECT, performed in our laboratory. ${ }^{5}$

There have been no published studies on changes in plasma prolactin following partial seizures. In his paper $^{2}$ Trimble states that he found no clear pattern in these patients. Apart from stating that two of his patients with partial seizures, one with partial complex epilepsy, had elevated levels, he gives no further details. Our findings suggest that plasma prolactin levels are unchanged following partial seizures with elementary symptomatology. We are unable to comment definitively on patients with complex partial epilepsy as only one of our patients had this type of epilepsy.

Our findings confirm that plasma prolactin levels are unchanged following pseudoseizures. This, and other studies ${ }^{23}$ would, therefore, suggest that esti- mation of plasma prolactin is useful in differentiating between generalised and pseudoseizures. The optimal time for this estimation is 15-20 minutes following a seizure.

The results of this study also suggest that prolactin levels are generally higher in patients with generalised epilepsy. This has been confirmed by others ${ }^{6}$ who have shown that prolactin levels are elevated in patients with idiopathic grand mal seizures without anticonvulsant therapy, and that short-term and long-term treatment with anticonvulsants further increase prolactin.

The mechanism by which generalised seizures induce a short lived elevation of plasma prolactin following seizures, is not known. Stress causes an elevation of plasma prolactin. However, this is unlikely to be the cause following seizures as it has been shown ${ }^{3}$ that, while serum cortisol rises following simulated seizures, prolactin is not significantly elevated. Our study confirms that whilst the stress of a pseudoseizure results in a slight rise in the prolactin level, as exemplified by a $C_{\max }$ of 200 at 20 mins. (table 3), this rise is not significant and the maximum prolactin level lies well within the normal range. Prolactin secretion is under predominantly inhibitory control from the hypothalamus. Dopamine appears to be the major inhibitory transmitter involved. It is possible that a rise in plasma prolactin will only occur when the abnormal electrical discharge in an epileptic seizure passes through the hypothalamic region. This might also explain why plasma prolactin levels are not elevated following partial seizures with elementary symptomatology.

Although other pituitary hormones have been shown to be elevated following ECT, there has been little published work on changes in these hormones following spontaneous seizures. Preliminary studies in our patients suggest that changes in growth hormone levels are very variable, and are not of benefit in distinguishing between generalised and pseudoseizures.

A preliminary report of this study was presented at the first regional meeting of the British-DanishDutch Branches of the International League against Epilepsy held in Heeze, The Netherlands, in October, 1981.

We thank the Medical Research Council of Ireland for financial support, and Dr J Seldrup for statistical advice.

\section{References}

' Meldrum BS, McWilliam JR. Hormone changes following seizures. In: Dam M, Gram L, Penry JK, eds. 
Advances in Epileptology: XIIth Epilepsy International Symposium. New York: Raven Press, 1981:441-8.

${ }^{2}$ Trimble MR. Serum prolactin in epilepsy and hysteria. Br Med J 1978;2:1682.

${ }^{3}$ Abbott RJ, Browning MCK, Davidson DLW. Serum prolactin and cortisol concentrations after grand mal seizures. J Neurol Neurosurg Psychiatry 1980;43:163-7.

${ }^{4}$ Lanigan O, Powell D. Human prolactin: Radioimmunoassay studies. Ir J Med Sci 1978;147:242-8.
${ }^{5}$ Skrabanek P, Balfe A, Webb M, Maguire J, Powell D. Electroconvulsive therapy (ECT) increases plasma growth hormone, prolactin, luteinising hormone and follicle-stimulating hormone, but not thyrotropin, or substance P. Psychoneuroendocrinology 1981; 6:261-7.

${ }^{6}$ Matz DR, Rolf LH, Sitzer G, Brune GG. Prolactin and growth hormone in idiopathic grand mal seizures. In: Dam M, Gram L, Penry JK, eds. Advances in Epileptology: XIIth Epilepsy International Symposium. New York: Raven Press, 1981: 477-81. 